\title{
Die einfache Form des Erzählens (Experimentelle Geisteswissenschaften)
}

Fritz Breithaupt ${ }^{1}$

Welches sind die Eigenschaften, die eine Geschichte zu einer Geschichte machen? Was ist die Grundform des Erzählens? Und was wird wiedergegeben, wenn Menschen eine kurze Geschichte weitererzählen?

Diese Fragen sind sicherlich zu groß für eine kleine Abhandlung. Dennoch können sie einen Leitfaden für eine Vielfalt möglicher Untersuchungen mit unterschiedlichen Verfahren und methodischen Prämissen darstellen. Dieser Beitrag untersucht mittels experimenteller Verfahren, was passiert, wenn Menschen kurze Geschichten nacherzählen. Untersucht wird dabei, was die Menschen beibehalten, was sie verändern und was sie fallenlassen. Dieses Verfahren soll Aufschluss geben über den minimalen Kern dessen, was wir erzählen. Im Mittelpunkt steht dabei die für viele Geisteswissenschaftler nicht unproblematische Frage der Wiederholbarkeit.

Die folgende Darstellung wird zunächst eine experimentelle Versuchsanordnung beschreiben und daraufhin die Ergebnisse skizzieren. Wenn man diese verallgemeinert, ergibt sich ein erstaunliches Bild von der einfachen Form des Erzählens. Anhand dieser Untersuchung werden abschließend einige Grundzüge der experimentellen Geisteswissenschaften vorgestellt und am Ende kurz das Problem der Wiederholbarkeit diskutiert.

Das Ergebnis wird deutlich ausfallen: Der Beitrag wird vorschlagen, dass Narration zumindest in den untersuchten Fällen als eine Affektkapsel verstanden werden sollte, also ein Transportmittel, das einen emotionalen Eindruck von einem Erzähler auf einen Hörer oder Leser überträgt. Das bemerkenswerte an Narration ist vielleicht eben dies, dass Erzählungen Affekte provozieren, die ein Hörer wiederum durch Weitererzählen relativ stabil weitergeben kann. Erhalten bleibt der Affekt und nicht unbedingt die Fakten, wer was mit wem gemacht hat. Narration ist insofern nicht die Abbildung einer Handlung, keine sachliche Instruktion und kein Mittel zur Vermehrung von Wissen. Narration ist auch keine Abbildung eines Affekts, sondern das performative Medium zur Herstellung des Affekts. Entsprechend kann in Hinblick auf das Erzählerische vieles wie etwa die Fakten der Erzählung verändert oder fallen gelassen werden, solange die Herstellung des Affekts gewährleistet wird. Die Stärke dieser Thesen ist natürlich bedingt durch die spezifische Perspektive auf die Frage des Erzählens und den experimentellen Versuchsaufbau.

Dieser Beitrag untersucht nur einen einzigen, allerdings für Narration sehr wichtigen Affekt, nämlich den der Überraschung. Es gibt aber gewisse Indizien dafür, dass diese Thesen auch für einige andere Affekte Geltung haben. Beginnen wir also am Anfang.

1 Ich danke Arne Willée für die kritische Lektüre dieses Beitrags, Michel Chaouli und Johannes Türk für ein anregendes Gespräch vorab und den Organisatoren und Teilnehmern des DFG-Symposiums für die reiche Diskussion. 
Das Erzählen und Verarbeiten von Geschichten ist mit Sicherheit eine zentrale kulturbildende Kraft, deren Bedeutung weit über das Literarische und Unterhaltende hinausgeht. ${ }^{2}$ Auch etwa der Klatsch, die religiösen Mythen und die Zeugenaussage gehören zum Erzählen. Doch was kennzeichnet es? Man darf sich fragen, ob es einige einfache Formen oder Grundtypen des Erzählens gibt, die vielfach variiert die Bausteine alles Erzählens darstellen. ${ }^{3}$ Oder ob der Vorzug des Erzählens gerade in seiner großen Dehnbarkeit und Komplexität besteht, die sich gerade nicht auf Grundtypen oder -formen festlegen lässt und an alle Ereignisse der wirklichen Welt anpassen kann?

Um sich der Frage einer möglichen einfachen Form des Erzählens zu nähern, wird in diesem Beitrag vor allem eine Eigenschaft von Erzählungen, Geschichten und Narrationen (zwischen denen wir in diesem Beitrag keinen Unterschied sehen müssen) untersucht: ihre Erzählbarkeit. Das scheint ein bescheidenes Mittel zu sein, um sich einem weiten und kulturell so wichtigen Phänomen wie der Narration zu nähern. Immerhin dürfen wir in der Tat annehmen, dass die Form der Narration sich in einem erheblichen Maße von ihrer Erzählbarkeit ableitet. Erzählbarkeit umfasst dabei, nahe an der Tautologie, zunächst einmal nichts anderes als die Vielfalt dessen, was tatsächlich erzählt wird. Eben diese Eigenschaft des Erzählbaren - dass es erzählt wird - soll experimentell genutzt werden. Diese Eigenschaft erlaubt es nämlich zu beobachten, was bei der Nacherzählung einer vorgegebenen Geschichte tatsächlich wiedergegeben und was fallengelassen wird. Auf diese Art und Weise wird die theoretische Frage der Definition der Erzählbarkeit in eine empirisch-experimentelle umgeformt, in deren Zentrum die Wiederholbarkeit steht.

Hier kann und muss man sich fragen, was das Verhältnis zwischen Erzählen und Nacherzählen ist. Kann das bloße Nacherzählen einer gegebenen Geschichte denn überhaupt Auskunft über das Erzählen geben? Ist das Nacherzählen nicht nur ein bloßer Akt der Erinnerung, während das Erzählen kreativ und produktiv ist? Erinnern wir uns zunächst daran, dass wir davon sprechen »ein Märchen zu erzählen «, wenn wir eigentlich meinen, dass wir ein uns bekanntes Märchen nacherzählen.

2 Siehe Albrecht Koschorke, Wahrheit und Erfindung: Grundzüge einer Allgemeinen Erzähltheorie, Frankfurt a. M. 2012. Die Leistungen von Geschichten sind für das intellektuelle Vermögen nicht wegzudenken. Geschichten stellen Dinge, Akteure, Orte, Handlungen und Geschehnisse in zeitliche und kausale Abfolgen, die erst darin übersehbar werden. Geschichten bündeln Information (wer was gemacht oder erlebt hat), produzieren Erklärungen und Ausreden, kommunizieren Wissen, machen die Positionen anderer Menschen zugänglich, erhalten und tradieren Vergangenes und bereiten dabei noch Vergnügen. Zugleich können Geschichten vielfach eingesetzt und manipuliert werden, um Institutionen zu stützen oder zu stürzen und um individuelle Positionen zu stärken oder zu schwächen.

3 Hierzu zählt etwa die ringförmige Heldengeschichte von Joseph Campbell, The hero with thousand faces [1949], Novato 2008. Die moderne Narratologie hat auch die kausale Minimalgeschichte von E. M. Foster (1927) bisweilen in den Status einer Grundform erhoben (»Der König starb, dann starb die Königin aus Trauer«). Hinter Wladimir Propps Morphologie des Märchens (München 1972 [1928]) erscheint ebenfalls eine Grundform vieler russischen Zaubermärchen. - Man denke etwa auch an die einfachen Formen von André Jolles, Einfache Formen. Legende, Sage, Mythe, Rätsel, Spruch, Kasus, Memorabile, Märchen, Witz, Halle (Saale) 1930; die sieben Standardformen von Christopher Booker, The seven basic plots: Why we tell stories, London/New York 2004; oder auch an die 36 dramatischen Situationen von Georges Polti, Les Trente-Six Situations Dramatiques, Paris 1895. 
Überhaupt ist das meiste Erzählen genau genommen ein Nacherzählen von bereits vorliegendem sprachlichen Material. Natürlich gibt es auch den Fall der ersten Erzeugung einer Geschichte. Etwa: »Erzähl doch mal, was Du heute gemacht hast? « Doch selbst in diesem Fall ist es durchaus möglich, dass der Antwortende mit dem gleichen Gefühl und ähnlichen kognitiven Verfahren wie bei der Nacherzählung reagiert, da er ja nacherzählt, was passiert ist, selbst wenn es vorher keine sprachliche Erzählung gab. Selbst das freie Fabulieren und Dichten orientiert sich wohl häufig an bekannten Mustern, Schemata, ${ }^{4}$ und bekannten Abfolgen. Im Grunde genommen wissen wir nicht genau, ob und inwiefern Erzählen und Nacherzählen strukturell oder kognitiv unterschieden werden können.

Statt dem ersten Erzählen vor jeder Nacherzählung einen Sonderstatus einzuräumen, könnte man daher genau umgekehrt annehmen, dass das Nacherzählen die maßgeblichen Eigenschaften des Erzählens aufweist. Diese Wette gehen wir im Folgenden ein, wenn wir beobachten, was Nacherzählungen betonen und was sie fallenlassen.

Was erzählen wir, wenn wir Geschichten nacherzählen? Was registrieren wir, wenn wir eine Geschichte hören oder lesen, was bleibt hängen und was geben wir weiter und wie? Spielen die faktischen Umstände einer Geschichte eine größere Rolle (wer was mit wem macht oder erlebt), und wenn ja, welche, oder aber die affektiven Eindrücke des Rezipienten (etwa Schreck, Freude, Miterleben und die Bewertungen der Ereignisse)? Orientieren wir uns an einer kleinen Zahl von zentralen Ereignissen oder aber an einer Vielzahl kleiner Details und ihrer Abfolge? Sind die Instruktionen und das vorgestellte Publikum für die Nacherzählung entscheidend? ${ }^{5}$ Um uns diesen Fragen zu nähern, stützen wir uns im Folgenden auf das empirische Verfahren der seriellen Reproduktion, welches 1932 von Frederic Bartlett entwickelt wurde. ${ }^{6}$

\section{Das Einfache als das Reproduzierbare}

Wenn Erzählbarkeit zum Ausgang der Untersuchung gewählt wird, gibt es eine einfache Konsequenz. Das Erzählbare ist das, was tatsächlich erzählt und nacherzählt wird. Man kann annehmen, dass das, was erzählt wird, bestimmte sprachliche, kulturelle und kognitive Eigenschaften aufweist, die es geeignet für Erinnerung und Wiedergabe machen. Insofern ist das Erzählte das `Einfache ‘ der Narration, denn es wird erinnert und weitergegeben.

4 Zum Schema in Nacherzählungen siehe Elizabeth Marsh, »Retelling is not the same as recalling: Implications for memory«, in: Current Directions in Psychological Science 16 (2007), $16-20$.

5 So haben Dudukovic, Marsh und Tversky festgestellt, dass Menschen, die zum Nacherzählen aufgefordert werden, die Geschichte deutlich verschieden nacherzählen und auch erinnern (!), je nachdem ob ihnen gesagt wurde, sie sollten >unterhalten oder sinformieren ‘; N. M. Dudukovic/E. J. Marsh/B. Tversk, »Telling a story or telling it straight: The effects of entertaining versus accurate retellings on memory«, in: Applied Cognitive Psychology 18 (2004), 125-143.

6 Siehe Frederic C. Bartlett, Remembering: An experimental and social study, Cambridge, Mass. 1932. 
Der Gegensatz des Einfachen ist dabei nicht das `Komplexe`, wie es der Titel des Sammelbands suggerieren könnte, sondern das, was nicht erzählt oder nacherzählt wird, also das Nicht-Erzählte, Entbehrliche, Vergessene. Das Nicht-Erzählte kann zwar durchaus fallengelassen werden, weil es zu kompliziert wirkt, aber es kann ebenso gut fallengelassen werden, weil es etwa uninteressant, sekundär, anstößig, schwierig für die Erinnerung, unpassend oder erzählerisch ungeeignet erscheint. Auch das Komplexe hat eine Rolle zu spielen, wie sich zeigen wird. Doch diese Komplexität taucht im Inneren des Erzählten auf, im Inneren der einfachen Form des Erzählens und ist mithin bereits Teil des `Einfachen`.

Im Folgenden werden wir uns auf einige Aspekte konzentrieren:

\section{Das Ereignis}

Es gibt wohl kaum eine Erzählung ohne Ereignis; und eine Narratologie ohne einen Begriff von Ereignis wäre keine. Drei unterschiedliche Ansätze zur Definition des >Ereignisses` können unterschieden werden. Computerlinguisten verwenden Ereignis unter anderem als Begriff für die einfachste sprachliche Sequenz mit Verb. Narrative Texte sind demgemäß Ketten von vielen Einzelereignissen. ${ }^{7}$ Narratologen definieren Ereignisse als Geschehnisse, die Situationen verändern und insofern eine kontextuelle Relevanz besitzen. Zu den Eigenschaften der narratologisch beobachteten Ereignisse gehören neben der Relevanz Unerwartetheit, Irreversibilität und Nicht-Wiederholbarkeit. ${ }^{8}$ Von Psychologen wird das Ereignis unter anderem als Raum einer kognitiv wahrgenommenen Einheit bemessen. Bestimmbar sind diese Ereignisräume von ihren Grenzen her. Anscheinend registrieren Menschen sehr genau, wann ein Ereignisraum beginnt und wo er endet. ${ }^{9}$

Im Folgenden werden wir uns auf einen - allerdings wichtigen - Fall von narratologisch relevantem Ereignis beschränken, nämlich den von Problem und Lösung bzw. von An- und Entspannung. ${ }^{10}$ Ein Ereignis in diesem Sinne liegt dann vor, wenn ein Problem, eine schwierige Situation oder eine Herausforderung für eine Person oder einen narrativen Charakter durch bestimmte Handlungen oder Geschehnisse gelöst oder transformiert wird.

\section{Die Überraschung}

Ereignisse sind unterschiedlich überraschend. Menschen im Allgemeinen und Rezipienten von Narrationen im Besonderen zeigen große Übereinstimmung in

7 Siehe Peter Hühn, »Event and eventfulness«, in: Ders./John Pier/Wolf Schmid u. a. (Hrsg.), Handbook of narratology, Berlin/New York 2009, 80-97. In Anlehnung an Propp siehe Mark A. Finlayson, Learning narrative structure from annotated folktales, $\mathrm{PhD}$ diss., Massachusetts Institute of Technology, Cambridge, Mass. 2012.

8 Vgl. Wolf Schmid, "Narrativity and eventfulness", in: Tom Kindt/Hans-Harald Müller (Hrsg.), What is narratology? Questions and answers regarding the status of a theory, Berlin/ New York 2003, 17-35; Wolf Schmid, Narratology. An introduction, Berlin/New York 2010.

9 Gabriel A. Radvansky/Jeffrey M. Zachs, Event recognition, Oxford 2014.

10 Vgl. Andrew Hamilton/Fritz Breithaupt, »These things called event: Toward a unified narrative theory of events«, in: Sprache und Datenverarbeitung 37 (2013), 65-87. 
der Bewertung des Grads an Überraschung eines Ereignisses. ${ }^{11}$ Überraschung ist allem Anschein nach ein zentrales und allgemein verständliches Element von Narration. ${ }^{12}$ Typischerweise enthält Überraschung zwei Merkmale: einerseits die emotionale Aufregung, also einen Affekt, ${ }^{13}$ und andererseits eine Verletzung von Wahrscheinlichkeit ${ }^{14}$ bzw. den Grad der Kluft zwischen Ursache und Wirkung. ${ }^{15}$ Für die narrative Überraschung spielen zudem Spannung, ${ }^{16}$ Wichtigkeit und Bedeutung, Versetzung in virtuelle Welten ${ }^{17}$ und Empathie ${ }^{18}$ eine Rolle. Für diese Studie ist es vor allem wichtig, dass Rezipienten den Grad an Überraschung eines Ereignisses anscheinend genau registrieren. Relevanz erlangt die Überraschung dabei als eine affektiv-emotionale Einstellung zur Problemlösung, die von der Entspannung bis zum Staunen und von der Freude bis zum Schock vielfache Formen annehmen kann. Überraschung kann dabei positiv oder negativ sein.

\section{Der Affekt}

Wenn Überraschung als Affekt, also als emotionale Reaktion aufgefasst wird, erlaubt diese Zuordnung einen Blick auf ein viel weiteres Phänomen, nämlich das der durch Narrationen im Rezipienten erweckten Affekte. Wir werden im Rahmen dieses kurzen Beitrags keine Gelegenheit haben, dieses Feld der narrativen Affekte und für

11 Vgl. R. Grimes-Maguire/M. T. Keane, "Expecting a surprise? The effect of expectations on perceived surprise in stories", in: Proceedings of the 27th Annual Conference of the Cognitive Science Society (2005), 833-838.

12 Für unsere Testpersonen war der Begriff der Überraschung verständlicher und er wurde genauer bewertet als der verwandte narratologische Begriff der Ereignishaftigkeit. Unter dem Begriff der Ereignishaftigkeit wird dabei auch die Lebhaftigkeit der Darstellung gefasst, was die Verständnisschwierigkeiten erklären könnte; siehe J. Graffam/J. L. Turner, »Escape from boredom: The meaning of eventfulness in the lives of clients at a sheltered workshop «, in: Robert B. Edgerton (Hrsg.), Lives in Process: Mildly Retarded Adults in a Large City, Washington, DC 1984, 121-144. Zum narratologischen Begriff der Ereignishaftigkeit siehe Schmid, Narratology (Anm. 8); Carola Gruber, Ereignisse in aller Kürze: Narratologische Untersuchungen zur Ereignishaftigkeit in Kürzestprosa von Thomas Bernhard, Ror Wolf und Helmut Heißenbüttel, Bielefeld 2014.

13 Vgl. J. K. Burgoon, »Interpersonal expectations, expectancy violations, and emotional communication", in: Journal of Language and Social Psychology 12 (1993), 30-48; K. H. Teigen/G. Keren, »Surprises: Low probabilities or high contrasts? «, in: Cognition 87 (2003), 55-71.

14 Vgl. Rainer Reisenzein, »Exploring the strength of association between components of emotion syndromes: The case of surprise«, in: Cognition \& Emotion 10 (2000), 241-277.

15 Vgl. R. Maguire/P. Maguire/M. T. Keane, »Making sense of surprise: An investigation of the factors influencing surprise judgments«, in: Journal of Experimental Psychology: Learning, Memory, and Cognition 37 (2011), 176-186; Daniel Kahneman/D. T. Miller, »Norm theory: Comparing reality to its alternatives«, in: Psychological Review 93 (1986), 136-153.

16 Vgl. Paul J. Silva, "Looking past pleasure: Anger, confusion, disgust, pride, surprise, and other unusual aesthetic emotions", in: Psychology of Aesthetics, Creativity, and the Arts 3 (2009), 48-51; Schmid, Narratology (Anm. 8); in der Musik: Elizabeth H. Margulis, On repeat: How music plays the mind, Oxford 2014.

17 Vgl. Melanie C. Green/J. K. Donahue, »Simulated worlds: Transportation into narratives«, in: Handbook of imagination and mental simulation, hrsg. Keith D. Markman/William M. P. Klein/Julie A. Suhr, New York 2009, 241-256.

18 Vgl. Susanne Keen, Empathy and the novel, Oxford/New York 2007. 
Narrationen relevanten Affekte adäquat abzustecken. ${ }^{19} \mathrm{Zu}$ ihnen zählen etwa Sympathie/Abneigung, Parteinahme, Wahrnehmung von Risiko, ${ }^{20}$ Furcht, Schreck, Ekel, Bevorzugung, ${ }^{21}$ Mitleiden und Rührung ${ }^{22}$. Auch das intuitive moralische Urteil und die Stimmung dürften in das Umfeld des Affekts gehören. ${ }^{23}$

\section{Lineare und ereignisgebundene Iteration}

Ausgehend von dem Akt des Nacherzählens können zwei Strategien des Nacherzählens unterschieden werden. Die eine Art begreift die Erzählung als einen Strang von Elementen, in der alle Elemente der Narration wie bei einer Perlenkette aufeinander folgen und in der die einzelnen Elemente aus den je früheren Elementen hervorgehen. Wer eine Geschichte nacherzählt, hangelt sich von einem Glied zum nächsten. Wird eines der Glieder vergessen oder verwechselt, kann die ganze Geschichte in eine andere Richtung gehen oder ihren Sinn gänzlich verlieren. Diese Form der Nacherzählung wird im Folgenden lineare Iteration genannt.

Die andere Strategie des Nacherzählens konstruiert Geschichten ausgehend von einigen zentralen Ereignissen. Jedes einzelne Element der Geschichte ist nur wichtig, insofern es mit einem der zentralen Ereignisse verbunden ist, zu ihm hinführt oder aus ihm hervorgeht. Das einzelne Element wird verändert oder weggelassen, je nachdem wie das Ereignis in einer neuen Nacherzählung ausfällt. Diese Strategie

19 Vgl. Christiane Voss, Narrative Emotionen. Eine Untersuchung über Möglichkeiten und Grenzen philosophischer Emotionstheorien, Berlin/New York 2004.

20 Vgl. Mehdi Moussaï/Henry Brighton/Wolfgang Gaissmaier, »The amplification of risk in experimental diffusion chains", in: Proceedings of the National Academy of Sciences 112/18 (2015), 5631-5636.

21 Vgl. David N. Rapp/Richard J. Gerrig, »Predilections for narrative outcomes: The impact of story contexts and reader preferences", in: Journal of Memory and Language 54 (2006), $54-67$.

22 Zur Rührung vgl. J. Hanich/V. Wagner/M. Shah u. a., »Why we like to watch sad films. The pleasure of being moved in aesthetic experiences«, in: Psychology of Aesthetics, Creativity, and the Arts 8 (2014), 130-143.

23 Viele Studien belegen, dass moralische Urteile eine deutlich intuitive Komponente haben und dass diese intuitiv-spontane Komponente gegenüber der rationalen Reflexion überwiegt. Einer der Vertreter einer Theorie des intuitiven (affekthaften) Urteils ist Jonathan Haidt, der fünf moralische Grundlagen (moral foundations) unterscheidet, vgl. Jonathan Haidt/Selin Kesebir, »Morality«, in: Susan T. Fiske/Daniel T. Gilbert/Gardner Lindzey (Hrsg.), Handbook of Social Psychology, Hoboken, NJ 52010, Bd. 2, 797-832. Diese intuitiven Urteile sind Affekten zumindest nahe verwandt, insofern sie im Rezipienten eine emotional-affektive Einstellung zur geschilderten oder erlebten Situation evozieren, die zahlreiche irrationale Verhaltensweisen mit einschließt. Ebenso wie die narrative Ereignishaftigkeit entsteht der moralische Affekt aus der Spannung zwischen einzelnen Elementen einer Narration. Im Falle moralischer Affekte gibt es eine Diskrepanz zu späteren Elementen: Wir erwarten oder hoffen, dass Unrecht später wieder gut gemacht oder bestraft wird oder eine gute Tat später belohnt wird. William Flesch geht so weit zu argumentieren, dass das Hauptgeschäft von Narration und Literatur in evolutionärer Perspektive im genauen Beobachten, Verfolgen und Bestrafen der Missetäter und ihrer Sympathisanten besteht; siehe William Flesch, Comeuppance. Costly signaling, altruistic punishment, and other biological components of fiction, Cambridge, Mass./London 2007. 

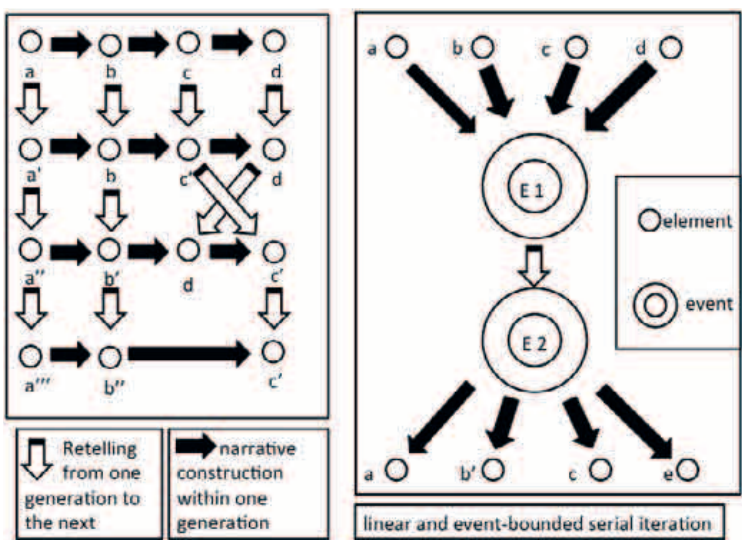

Abb. 1

der Nacherzählung wird im Folgenden ereignisgebundene Iteration (event-bounded iteration) genannt (Abb.1).

Man kann davon ausgehen, dass die meisten Nacherzählungen beide Strategien kombinieren. Fraglich ist, ob diese Unterscheidung bereits für den Akt des Erzählens gilt. In der Tat kann man sich gut vorstellen, wie das Erzählen als ein Springen von Punkt zu Punkt verläuft oder aber gezielt auf einige große Ereignisse zielt. Hinter dieser Unterscheidung steht die Frage, ob eine Geschichte durch einige große Ereignisse strukturiert wird oder besser als eine Abfolge charakterisiert wird.

\section{Verfahren der Nacherzählung (Beschreibung der Experimente)}

Mit den im Folgenden vorgestellten Experimenten sollte ermittelt werden, ob Überraschung bei Nacherzählungen eine wichtige Rolle spielt und ob es gegebenenfalls einen optimalen Grad von Überraschung gibt, der zu besseren Nacherzählungen führt. Dahinter steht die Frage, welche Rolle Überraschung für die einfache Form des Erzählens spielen könnte. Bevor wir die Ergebnisse vorstellen, ist es wichtig, das experimentelle Verfahren der Nacherzählung kurz darzustellen, denn natürlich prägen die Versuchsanordnungen die Resultate. Genauere Übersichten zum Verfahren finden sich in den zitierten Arbeiten. ${ }^{24}$

In den Versuchen erzählen Freiwillige in einem Stille-Post-Spiel schriftlich kurze Geschichten nach. Jede Einzelgeschichte wird dabei von je drei verschiedenen Nacherzählern hintereinander nacherzählt, wobei der jeweils nachfolgende Erzähler nur

24 Vgl. Yoshihisa Kashima, »Maintaining cultural stereotypes in the serial reproduction of narratives", in: Personality and Social Psychology Bulletin 26/5 (2000), 594-604; Yoshihisa Kashima/Victoria Wai-Lan Yeung, »Serial reproduction: An experimental simulation of cultural dynamics«, in: Acta Psychologica Sinica 42/1 (2010), 56-71; Ara Norenzayan/Scott Atran/ Jason Faulkner u. a., "Memory and mystery: The cultural selection of minimally counterintuitive narratives«, in: Cognitive Science 30/3 (2006), 531-553; J. L Barrett/M. A. Nyhof, "Spreading non-natural concepts: The role of intuitive conceptual structures in memory transmission of cultural materials«, in: Journal of Cognition and Culture 1 (2001), 69-100. 
die Geschichte seines unmittelbaren Vorgängers erhält.. ${ }^{25}$ Dies wird bei jeder Ausgangsgeschichte vielfach wiederholt. Dann werden alle Ergebnisse der dritten Nacherzählung, wiederum von anderen Freiwilligen, auf den Grad ihrer Überraschung hin ausgewertet und verglichen.

Die Teilnehmer der hier vorgestellten Studien wurden bisher über die elektronische Plattform Mechanical Turk rekrutiert. ${ }^{26}$ Die einzigen Vorgaben waren, dass die Teilnehmer über 18 Jahre alt sein und in den USA leben mussten. Jeder Teilnehmer bekam die Anweisung, kurze Geschichten »in eigenen Worten« schriftlich nachzuerzählen. ${ }^{27}$ Die Geschichten erschienen dabei auf dem Bildschirm und konnten fast beliebig lange gelesen werden, bis der Teilnehmer sie auf einer nachfolgenden Seite wiedergab. Teilnehmer, die zu einem früheren Zeitpunkt an einer der Studien teilgenommen hatten, wurden per Filter von der weiteren Teilnahme ausgeschlossen. Die Teilnehmer unserer Studien waren im Durchschnitt 30 bis 35 Jahre alt und hatten einen sehr unterschiedlichen Bildungshintergrund. ${ }^{28}$ Die Studie aus meinem Arbeitskreis konnte auch eine viel größere Zahl an Nacherzählungen berücksichtigen (1131 in Studie 1 mit kurzen Ausgangserzählungen und 855 in Studie 2 mit längeren Ausgangstexten). Insgesamt waren 3299 Freiwillige an der folgenden, hier nur kurz zusammengefassten Studie beteiligt.

Die den Teilnehmern vorgelegten Ausgangsgeschichten schildern je einen Charakter in einer problematischen Situation. Ein Student etwa ist einer Abschlussprüfung nicht gewachsen, eine Tochter hat einen Streit mit ihrer Mutter oder ein Schüler ist zu schüchtern, um ein Mädchen anzusprechen. Dann passiert etwas, und die problematische Situation wird dadurch aufgelöst. Was aber passiert, variiert. Für jede der drei Ausgangsgeschichten wurden je sieben verschiedene Ereignisse und also Variationen konstruiert. Einige Varianten spielen sich im erwarteten Bereich $\mathrm{ab}$, andere grenzen an große Unwahrscheinlichkeit.

Exemplarisch seien hier einige der Ausgangstexte wiedergegeben. Variiert wurde je die mit »XXX« markierte Stelle, wie mittels einiger Beispiele angedeutet werden soll. In eckigen Klammern ist der von vielen Teilnehmern ermittelte mittlere Wert (Median) von Überraschung angegeben. Dieser Wert wurde bestimmt, indem eine große Gruppe von Freiwilligen je 15 der insgesamt 21 Variationen in vollständig

25 Die dreimalige Nacherzählung ist auch von Barrett und Nyhof favorisiert worden, siehe J. L. Barrett/M. A. Nyhof, "Spreading non-natural concepts: The role of intuitive conceptual structures in memory transmission of cultural materials«, in: Journal of Cognition and Culture 1 (2001), 69-100. Unsere Versuche haben ebenfalls ergeben, dass nach drei Nacherzählungen eine relative Stabilität erreicht ist.

26 Vgl. ausführlicher zu den hier vorgestellten Versuchen und Ergebnissen: Fritz Breithaupt/ Binyan Li/Eleanor Brower u. a., "Serial reproduction of narratives: Surprise beats facts in event retelling« [in Vorbereitung].

27 Diese Anweisung geht zurück auf Yoshihisa Kashima, »Maintaining cultural stereotypes in the serial reproduction of narratives", in: Personality and Social Psychology Bulletin 26/5 (2000), 594-604.

28 Die wichtige frühere Studie von Norenzayan, Altran, Faulkner und Schaller (Anm. 24) verwendeten ähnliche Verfahren der Nacherzählung, haben ihre Teilnehmer allerdings aus Studenten amerikanischer Universitätskurse rekrutiert und insofern eine viel homogenere Gruppe von Teilnehmern angesprochen. Zudem benutzte diese Studie keine differenzierten Grade an Überraschung. 
randomisierter Reihenfolge auf den Grad ihrer Überraschung auf einer Skala von 0 bis 7 hin bewertet hat.

Jason liked a girl in his class. He was very shy, however, and was too afraid to talk to her. One day, XXX. He mumbled that she looked nice and asked her if she would like to eat lunch with him.

- they were standing next to each other in a line (Jason A [1.05])

- as he was walking down the hallway he saw the girl and noticed that they had on the same outfit (Jason B [2.5])

- as he was walking behind her on the crosswalk to school, he noticed that a car was coming very fast towards them. He quickly ran and pushed her out of the way into safety (Jason G [6])

Robert sat down in class to take his final exam. He knew the exam would be difficult, but he was shocked to see how hard it was. He may not have studied enough, but this exam was simply not fair and he started sweating. With an hour left, he asked for a bathroom break and left the room. In the bathroom, XXX. Then he returned to the testing room to complete the exam.

- he splashed his face with water (Robert A [0.45])

- he found an envelope with his name on it. Inside was the answer key, signed "with love« from his teacher (Robert G [6.3])

In der zweiten Studie wurden die gleichen Geschichten um eine relativ ereignislose Vorgeschichte verlängert. ${ }^{29}$ Die Ausgangsgeschichten in Studie 2 umfassen dabei je 12 bis 15 Sätze. Die variierenden Elemente mit unterschiedlichen Überraschungsgraden beschränken sich dabei weiterhin auf die identischen 1 bis 2 Sätze. ${ }^{30}$

Eine der Absichten der Experimente besteht darin zu ermitteln, ob es einen genau erfassbaren optimalen Grad von Überraschung gibt, der zu einer besseren und genaueren Nacherzählung führt. Erkennbar wäre ein solcher optimaler Grad an Überraschung durch größere Stabilität des Sprachmaterials und der Fakten, der Länge und Qualität der Erzählung, sowie einer stabilen Übertragung der Überraschung von einer Generation der Nacherzählung zur nächsten.

\section{Ergebnisse der Experimente}

Die Studien befinden sich in Vorbereitung zur Publikation und sind daher noch nicht vollständig abgeschlossen. Dennoch zeichnen sich einige sehr deutliche Tendenzen ab. Das erste interessante Ergebnis besteht darin, dass der Grad der Überraschung der Ausgangsgeschichten und der dritten Nacherzählungen sehr ähnlich

29 Vgl. Breithaupt/Binyan/Brower u. a. (Anm. 26).

30 Die frühere Studie von Norenzayan, Atran, Faulkner und Schaller (Anm. 24) hatte, mit etwas anderen Zielen und Definitionen, ermittelt, dass bestimmte Konstellationen von Ereignishäufung zu besseren Nacherzählungen führen als andere. Das Ergebnis dass in sofortigen Nacherzählungen die Geschichten mit vielen kontrainduktiven En ssen am besten nacherzählt wurden, dass aber auf längere Sicht Geschichten mit einigen, aber nicht $\mathrm{zu}$ vielen kontrainduktiven Ereignissen am genausten erinnert wurden. 


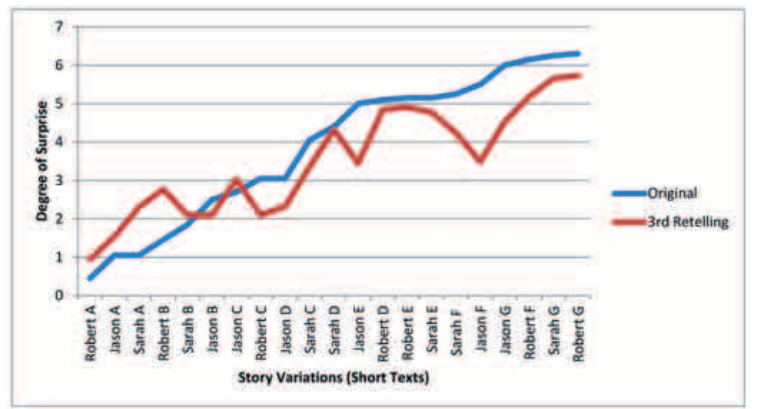

Abb. 2: Der Graph zeigt die Korrelation der mittleren Ereignishaftigkeit der kurzen Ausgangsgeschichten (also etwa Jason B) zu der mittleren Ereignishaftigkeit der dritten Nacherzählungen. Die Korrelation ist sehr stark $(r=0.95)$, trotz massiver Veränderungen der Ausgangsgeschichten. Quelle: Abbildung und Erläuterungen aus Fritz Breithaupt/Binyan Li/Eleanor Brower u. a., "Serial reproduction of narratives: Surprise beats facts in event retelling" [in Vorbereitung].

war, auch und obwohl die resultierenden Geschichten typischerweise um $90 \%$ (bei den langen Ausgangsgeschichten) geschrumpft waren. Dieser Effekt stellte sich weitgehend unabhängig vom Grad der Überraschung der Ausgangsgeschichten ein. Es finden sich unter den dritten Nacherzählungen also sehr viele Geschichten, die von den Bewertenden als ebenso überraschend wie die Ausgangsgeschichten eingestuft wurden; zugleich zeigten die Ausgangsgeschichten und die resultierenden Nacherzählungen deutliche Veränderungen in den Fakten, Wörtern und der Länge. Im Folgenden sollen die wichtigsten Ergebnisse kurz skizziert werden (die Studien werden bald vollständig publiziert).

Die genaue Korrelation des Überraschungsgrads und der dritten Nacherzählung zur Vorlage ist höchst beeindruckend. Bei den kurzen Geschichten ist die Pearson Korrelation (r) 0.93 bis 0.95 (je nach Ausgangsgeschichte), bei den langen Geschichten immerhin noch 0.69 bis 0.93 . Demgegenüber ist die Stabilität des Ereignisses gemessen an verschiedenen Faktoren wie Kernwörtern, Namen und Expertenlektüren aller Texte relativ schwach, vor allem bei den längeren Ausgangsgeschichten. Abb. 2 zeigt die Korrelation des Überraschungsgrads für die kurzen Ausgangsgeschichten.

Hier sind zwei Einzelergebnisse:

\section{Ergebnis 1}

Originalgeschichte:

Robert sat down in class to take his final exam. He knew the exam would be difficult, but he was shocked to see how hard it was. He may not have studied enough, but this exam was simply not fair and he started sweating. With an hour left, he asked for a bathroom break and left the room. In the bathroom, he loudly asked the exam question to the mirror and a voice gave him the answer. Then he returned to the testing room to complete the exam. [Bewertet als 6.15]

Dritte Wiederholung (Codename Robert 147): 
A boy named Robert was taking an exam. He went through the pages and noticed that he didn't know any of the answers. He asked to go to the restroom and while he was there he asked the questions to himself in the mirror. His reflection in the mirror told him the answers and he was scared. [Bewertet als 6]

Aus dem Rätsel, wer die Antwort gibt, folgt nun eine deutliche Antwort, deren Unheimlichkeit aber wiederum der affektiven Stärke der Ausgangsgeschichte ähnelt.

\section{Ergebnis 2}

Originalgeschichte:

Jason liked a girl in his class. He was very shy, however, and was too afraid to talk to her. One day, as he was walking down the hallway he saw the girl and noticed that they had on the same outfit. He mumbled that she looked nice and asked her if she would like to eat lunch with him. [Bewertet als 3.05]

Dritte Wiederholung (Codename Jason 58):

After a long time of admiration, Jason worked up the courage to ask his classmate out after noticing she was wearing the same outfit as him. After a short pause, she agreed. [Bewertet als 2.9]

Wie in den meisten Wiederholungen der kurzen Geschichten ist das zentrale Detail der gleichen Kleidung bewahrt, doch nun wird das Drama der Schüchternheit (Jason) gemildert, während zugleich das innere Drama der Reaktion des Mädchens in Szene gesetzt wird ([...] »after a short pause« $[\ldots])$.

Besonders auffällig ist das Resultat bei den längeren Geschichten. Bei den Ausgangsgeschichten variieren nur 1 bis 2 Sätze, während die übrigen 11 bis 14 Sätze identisch sind. In der Bewertung des Überraschungsgrads variieren die Ausgangsversionen in etwa entsprechend den kürzeren Texten (nur die Differenz zwischen den Geschichten mit niedrigem Überraschungsgrad [0-2] verschwindet). Daraus kann man schließen, dass die Bewertenden des Überraschungsgrads der Ausgangsgeschichten in der Tat auf eben das je unterschiedliche Ende zielten. Das Ergebnis der Nacherzählung fällt ähnlich wie bei der Studie mit kurzen Texten aus: Der Grad der Überraschung bleibt zwischen Ausgangsgeschichte und dritter Nacherzählung relativ stabil, doch die Ereignisse werden variiert oder verschwinden ganz.

Nun kommen wir zu dem zweiten, wirklich erstaunlichen Ergebnis. Nur 50,4\% der dritten Nacherzählungen erhielten das Ereignis in den Grundzügen, 18,9\% bewahrten Teile des Ereignisses, bei 30,7\% erscheint es nicht einmal im Ansatz. Erstaunlich daran ist, dass sich der Überraschungsgrad ursprünglich just auf eben dieses häufig verloren gegangene Ereignis gestützt haben muss, aber nun dennoch in den meisten Fällen erhalten bleibt. Das heißt, dass auch viele der Nacherzählungen, die das je überraschende Ereignis fallengelassen haben, den mit dem Ereignis verbundenen Überraschungsgrad bewahrt und auch ohne das ursprüngliche Ereignis weitergegeben haben, denn sonst wäre die hohe Korrelation der Überraschungswerte der Originalgeschichte und der dritten Nacherzählung nicht zu erklären. Ent- 
weder wurde das ursprüngliche Ereignis in den drei Nacherzählungen also bis zur Unkenntlichkeit verändert oder aber die Überraschung ging auf ein ursprünglich nebensächliches Ereignis über.

Ereignisse stehen in der Nacherzählung im Vordergrund. Doch diese Ereignisse werden nicht stabil tradiert, sondern als Träger eines spezifischen Überraschungsgrads bewahrt. Das erste Ergebnis spricht also deutlich für ereignisgebundene Iteration und für eine Erhaltung eines jeden Grads der Überraschung. Die Vermutung eines optimalen Überraschungsgrads hat sich also nicht bestätigt. Nur die extremen Überraschungsgrade von 0 bis 2 und 6 bis 7 werden anscheinend ungenauer weitergegeben.

Doch besonders interessant ist da. ite Ergebnis: Bewahrt wird der Grad der Überraschung, auch dann, wenn das Ereignis, das diese Überraschung ursprünglich auslöste, verschwindet $ه$ ist hier passiert und welche Schlüsse können wir für die Narration als ganze zienerr?

\section{Auswertung und Interpretation: Was ist die einfache Form der Erzählung?}

Wenn wir diese Ergebnisse weiterdenken - und sich die Ergebnisse in Folgeuntersuchungen verfestigen -, ergibt sich ein erstaunliches Bild. Das zentral wiedergegebene und nacherzählte Element einer Narration ist weniger die Handlungsabfolge als vielmehr der affektive Eindruck der Überraschung, den die Ereignisse einer Geschichte hinterlassen. Die Handlungsabfolge (plot) spielt natürlich auch eine wichtige Rolle, es sind aber d D eignisse, die den affektiven Eindruck hervorrufen, selbst wenn die Ereignisse in den Nacherzählungen variiert werden. Der spezifische Affekt, also der Grad der Überraschung, gibt das Paradigma vor, aufgrund dessen die Ereignisse variiert werden können (vgl. Abb. 3).

Wir können uns diese Sequenzen der Nacherzählung in diesem Experiment mithin als einen generativen Prozess vorstellen. Ein vorliegendes Ereignis wird verarbeitet, indem es auf einen Affekt zugespitzt wird. In der Nacherzählung wird aus diesem Affekt wiederum ein ihm entsprechendes Ereignis konstruiert. Das neue Ereignis enthält dabei zugleich eine Vielzahl an variierenden Versatzstücken und Einzelelementen des ersten Ereignisses (etwa: Personen, Handlungen, Situation, Ort, Details, Objekte, Instrumente, direkte Rede, etc.) sowie wichtige Schemata des Originals wie »Student unter Druck« und dergleichen. ${ }^{31}$ Doch der Affekt der Überraschung dominiert den konkreten Handlungsablauf des Ereignisses. Wenn die Nacherzählung wiederholt wird, kann eine Vielfalt von verschiedenen Einzelereignissen oder Narrationselementen erzeugt werden. Konstant bleibt nur der je erzeugte Affekt.

Affekte wie Überraschung können nun, anders als bloße Fakten, nicht einfach nacherzählt werden. Sie werden vielmehr zum Ziel des Nacherzählens, also zur Leitvorstellung dessen, was der Erzähler im Hörer bewirken will.

31 Vgl. Elizabeth J. Marsh, «Retelling is not the same as recalling: Implications for memory«, in: Current Directions in Psychological Science 16 (2007), 16-20. 

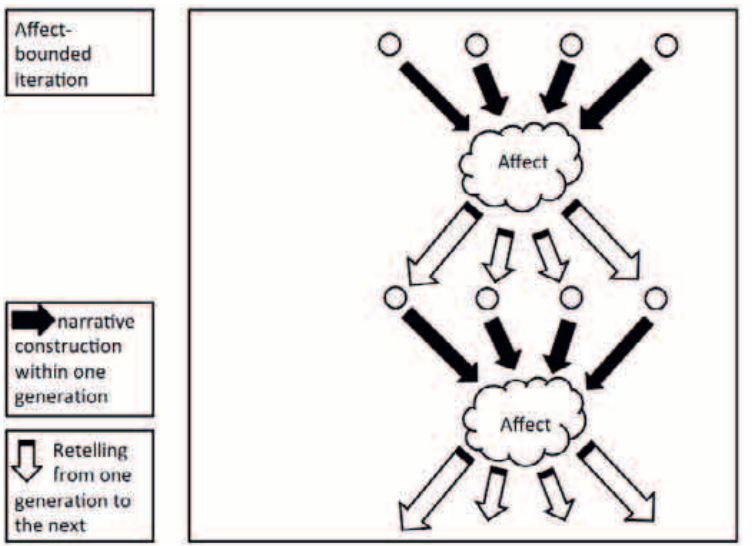

Abb. 3

Aus diesen Versuchsreihen sollen nun zwei teilweise spekulative Schlussfolgerungen gezogen werden. Die erste betrifft die Relevanz von Affekten wie die Überraschung für Nacherzählungen im Allgemeinen, die zweite die einfache Form der Narration.

Wenden wir uns also zunächst der Rolle der Affekte zu. Wenn es sich in der Tat bestätigt, dass eine ähnlich exponierte Rolle auch für andere narrative Affekte neben der Überraschung gilt - was wir bisher nicht wissen und bestenfalls vermuten können - dann werden auch dort die Ereignisse, und also auch die Fakten, den Affekten tendenziell unter- oder nebengeordnet. Wir haben den Begriff des narrativen Affekts oben sehr weit gefasst, was uns erlaubt auch Phänomene wie die Parteinahme, das intuitive Urteil und Empathie mit in den Blick zu nehmen. Allerdings besteht die Gefahr eines weitgefassten Begriffs natürlich darin, dass wir zu weit schauen und manches über einen Kamm scheren, was nicht zueinander gehört. Für jeden möglichen Affekt müssen natürlich erste entsprechende empirische Daten gesammelt werden.

Zumindest vom intuitiven Urteil wissen wir, dass es in Verfahren der wiederholten Nacherzählung ebenfalls beibehalten und unter Umständen verstärkt wird. ${ }^{32}$ Dies entspricht etwa einem häufig beobachteten Phänomen, wenn Kinder einen kritischen Fall einer moralisch suspekten Handlung wiedergeben. Sie verändern die eigentlichen Fakten und scheinen zu lügen. Doch vielleicht lügen sie hier viel weniger, als dass sie den bei ihnen festgesetzten affektiven Eindruck - A ist unschuldig oder $\mathrm{B}$ ist nicht zu trauen - adäquat wiedergeben. Dem Affekt gegenüber sind die Fakten tendenziell untergeordnet und können entsprechend verändert werden, solange sie mit dem Affekt korrespondieren, ohne dass sich das Gefühl einer Unwahrheit einstellt. Auch in diesem Falle würde das Erzählen und Weitererzählen entsprechend der Befunde dieser Arbeit als affektgebunden stattfinden.

32 Vgl. Anthony Lyons/Yoshihisa Kashima, »Maintaining stereotypes in communication: Investigating memory biases and coherence-seeking in storytelling ", in: Asian Journal of Social Psychology 9/1 (2006), 59-71. 
Natürlich erlernen Menschen, Fakten zu erinnern. ${ }^{33}$ Dennoch darf man sich fragen, in welchem Grade etwa Zeugen ähnlich verfahren. Sie haben einen intuitiven Eindruck einer Situation und eben diesen intuitiven Eindruck wollen sie in der Wiedergabe der Ereignisse bewahren. Entsprechend werden die Fakten diesen intuitiven (also affektiven) Eindrücken nachgeordnet. Dies muss nicht direkt in Lügen münden, kann aber mittels Betonung, Auslassung und Entfaltung zu faktischen Verzerrungen führen.

Auch im Falle des Risikos wurden Privilegierungen im Rahmen von serieller Reproduktion beobachtet. ${ }^{34}$ Zusammengenommen spricht also einiges für die zentrale Rolle von Affekten bei der Konstruktion und Rekonstruktion von Narrationen.

Kommen wir zurück zu unserer Ausgangsfrage der einfachen Form der Narration. Wir haben bisher das Verfahren der Nacherzählung in den Vordergrund gestellt. Die uns dabei leitende Annahme war, dass Erzählung und Nacherzählung derart strukturverwandt sind, dass Aussagen über die einfache Form der Erzählung gemacht werden können. Dabei geraten wir in eine gewisse Verlegenheit. Denn statt einen optimalen Grad von Überraschung festgestellt zu haben, haben wir beobachtet, dass fast jeder Grad von Überraschung genau registriert und weitererzählt wird. Insofern können wir eben nicht eine bestimmte Form des Ereignisses in den Mittepunkt stellen und als optimale oder auch einfache Form von Narration deklarieren.

Doch eben diese Einsicht erlaubt uns nun eine andere, viel deutlichere These über die einfache Form der Narration müssen uns von dem Gedanken befreien, dass eine einfache Form der Narration meiner textuell kodierten Handlungssequenz besteht. Stattdessen müssen wir das Primat oder zumindest einen zentralen Stellenwert des Affektiven einräumen. Die einfache Form der Narration ist dem entsprechend eine temporal-sequentielle Form, in der bestimmte Affekte erzeugt, aufbewahrt und perpetuiert werden. Um eine temporal-sequentielle Form handelt es sich, insofern alle Elemente einer Narration in zeitlichen Abfolgen dargestellt werden. Affekte wie die Überraschung wären anders nicht zu denken. Narrationen stellen mithin Affektkapseln dar. ${ }^{35}$

$\mathrm{Ob}$ die evolutionäre Funktion von Narrationen aber in der Aufbewahrung von Affekten besteht oder umgekehrt die Affekte die Funktion haben, eine narrative

$33 \mathrm{Zu}$ den Verzerrungen, etwa durch den sogenannten Flashbulb Effect, siehe: H. Welzer, »Renarrations: How pasts change in conversational remembering«, in: Memory studies 3 (2010), 5-17; S.-Å. Christianson, »Emotional stress and eyewitness memory: A critical review«, in: Psychological Bulletin 112 (1992), 284-309; A. Koriat/M. Goldsmith/A. Pansky, »Toward a psychology of memory accuracy«, in: Annual Review of Psychology 51 (2000), 481-537.

34 Vgl. Mehdi Moussaïd/Henry Brighton/Wolfgang Gaissmaier, »The amplification of risk in experimental diffusion chains«, in: Proceedings of the National Academy of Sciences 112/18 (2015), 5631-5636.

35 Eine Anmerkung in eigener Sache sei hier erlaubt. In Kultur der Ausrede habe ich vorgeschlagen, dass Narrationen sich dadurch ausweisen, dass sie im Rezipienten eine Vielheit von imaginären Versionen auslösen. Narratives Denken ist das Denken in Vielversionalität. Dieser Vorschlag wird in dem vorliegenden Beitrag durch den Begriff des Affekts insofern ergänzt, als Affekte die Erwartung einer Auflösung, Entwicklung oder Anerkennung bewirken. Wo eine Erwartung ist, dort gibt es Pluralität an Möglichkeiten. Siehe Fritz Breithaupt, Kultur der Ausrede. Eine Erzähltheorie, Berlin 2012. 
Situation besser in der Erinnerung zu verankern, ist hier nicht zu bestimmen. Selbst wenn die Evolutionsbiologie hier eine deutliche Antwort geben könnte, so dürfen wir annehmen, dass beide Möglichkeiten in der kulturellen Praxis derart eng miteinander verzahnt sind, dass sie nicht voneinander zu trennen sind.

Die einfache Form der Narration, so die These, ist die der Affektkapsel. Im Falle der hier vorgestellten Studien zur Überraschung resultiert der Affekt aus dem Grad an emotionaler Reaktion auf eine mehr oder weniger erwartete oder unerwartete Auflösung einer problematischen Situation. Im Falle der moralischen Intuition etwa bewahrt die Narration den Grad der Empörung über das Missverhältnis und schreibt sie als Erwartung von Belohnung/Strafe fort. Im Falle des Risikos wird die Warnung transportiert.

Aus der Perspektive dieser Studien erscheint es so, als hätten eine Reihe von vergangenen empirischen Studien zur Narration andere und vereinfachte Begriffe von Narration verwendet. Nicht jede sprachliche Abbildung einer Handlung ist schon an sich narrativ. Eine Narration liegt dann vor, wenn eine sprachliche oder sprachähnliche Darstellung einer Handlung oder von Charaktere betreffenden Geschehnissen einen Affekt im Rezipienten hervorruft, durch den die dargestellte Handlung zu einer kognitiven Einheit (oder einer Ansammlung von Einheiten) wird. $^{36}$

Hier kommen wir zurück zu der Frage von Einfachheit und Komplexität. Die einfache Form einer Narration verstanden als Affektkapsel weist eine erstaunliche Komplexität auf. Mit jedem narrativ aufbewahrten Affekt wird eine Vielfalt von Einzelerzählungen möglich, deren Paradigma in dem Affekt vorgegeben ist (etwa als spezifischer Grad an Überraschung, der allen Einzelerzählungen gemeinsam ist). In der Abfolge von Nacherzählungen könnte eine große Anzahl an Geschichten entstehen, ähnlich wie wir uns vorstellen können, dass aus Propps Grundtyp des russischen Zaubermärchens eine Vielzahl wurde, doch der bestimmende Affekt als das narrative Paradigma bleibt relativ konstant. Mannigfaltigkeit und Komplexität stehen der einfachen Form nicht entgegen, sondern werden von ihr ermöglicht. Goethe und nachfolgend Propp haben für diese Struktur von Komplexität als das Ineinander von Einzelnem und Allgemeinem den Begriff der Morphologie geprägt. $^{37}$

Es wird häufig argumentiert und belegt, dass Narration besonders gut für Erinnerung geeignet ist. Unsere Ergebnisse deuten an, dass emotionale Erinnerung und Weitergabe emotionaler Eindrücke dabei einen besonderen Stellenwert haben dürften. Daraus folgt zwar nicht direkt, kann aber vermutet werden, dass die menschliche Entwicklung emotionaler Differenzierungen durchaus mit Erzählvermögen

36 Mit der Idee der Einheit soll an die Gedankengänge und Studien von Radvansky untu Lachs angeschlossen werden; siehe Gabriel A. Radvansky/Jeffrey M. Zachs, Event cognition, Oxford 2014

37 Beginnend mit Texten wie »Der Versuch als Mittler 2 rorr bbjekt und Subjekt« (1792/1823) hat Goethe die Aufgabe des Beobachters in der Teilung des je Einzelnen und des je Allgemeinen beschrieben, also etwa der Einzelpflanze und der Urpflanze. Seine Einsicht war dabei, dass diese Teilung nie mit letzter Gewissheit gezogen werden kann, sondern stets ein Akt des Beobachters bleibt, da Einzelnes und Allgemeines stets nur in ein und derselben Gestalt erscheinen. 
und Narration gekoppelt ist. Narrationen, so die Vermutung, trainieren uns, Emotionen zu erkennen und korrekt zu bewahren, auch wenn uns für sie das adäquate Vokabular zur Benennung fehlt. Affekt ist nicht gleich Affekt, und die Verfeinerung der menschlichen Sensorien für Emotionen mittels Narration dürfte wesentlich zu den komplexen sozialen Strukturen unserer Gesellschaft beitragen.

\section{Methodisches zu den experimentellen Geisteswissenschaften}

Abschließend sei eine kurze Anmerkung zur Methode angefügt, das heißt zu dem, was hier experimentelle Geisteswissenschaften genannt wird. Dieser Beitrag stellt die Ergebnisse einiger Experimente vor, die mit den typischen Standards der Kognitionswissenschaften und Psychologie durchgeführt wurden. Zu den zentralen Merkmalen gehören dabei die Wiederholbarkeit des Versuchs, die große Zahl an Probanden und die statistische Signifikanz. Die Fragestellung und das Interesse an der Narration dagegen stammt aus den Geisteswissenschaften. Man darf sich fragen, was experimentelle und empirische Geisteswissenschaften leisten können, was ihre Grenzen sind und wie sie sich zu den klassischen Geisteswissenschaften verhalten? Polemischer gefragt - und ich hoffe hier den kritischen Impetus meiner Diskussionspartner aufzunehmen ${ }^{38}$-: Können empirische Befunde einem Geisteswissenschaftler überhaupt etwas Interessantes sagen?

Wiederholbarkeit bedeutet, dass man das gleiche Ergebnis erwarten kann, sofern alle relevanten Umstände gleich sind, egal wer Experiment wo und wann wiederholt. Oberflächlich betrachtet scheint Wiede barkeit mithin vielen Prinzipien der Geisteswissenschaften zu wiedersprechen, also etwa Singularität, Interpretation, Subjektivität, Geschichtlichkeit und Kultur. ${ }^{39}$ Hier sollen zwar nicht ungebrochen die Charakterisierungen der Natur- und Geisteswissenschaften des neunzehnten Jahrhunderts perpetuiert werden, aber in einem erstaunlichen Maße gelten viele dieser Normierungen auch heute noch.

Dennoch wäre es ein Kurzschluss, das empirisch Wiederholbare schlicht als Gegensatz zum `Geisteswissenschaftlichen` anzusehen. Damit Singuläres, Einzelnes, Interpretation, Subjektives, Geschichtliches oder Kulturelles zum Vorschein kommen können, bedarf es fester, allgemeiner und also wiederholbarer Rahmen-

38 Der Beitrag wird hier weitgehend in der Fassung der Diskussionsvorlage zum DFG-Symposion »Komplexität und Einfachheit« im September 2015 wiedergegeben; ergänzt wurden nur diese kurzen Nachbemerkungen. Widerstand schien der Beitrag weniger hinsichtlich seiner Ergebnisse, als vielmehr seines Vorgehens auf den Plan zu rufen.

39 Natürlich gibt es vielfältige Formen, in denen die geisteswissenschaftlichen Ansätze sich dem Singulären zuwenden. Es kann als Einzelfall bzw. als Ausnahme oder Kasus (im Sinne von André Jolles) auftreten, die bzw. der dem Wiederholbaren gegenübersteht. Es ist auch in einer Interpretation zu finden, die hermeneutisch alle Daten eines Kunstwerks zu berücksichtigen hat und in eine einzigartige Konstellation bringt. Das Singuläre kann zudem in dem politischen Standpunkt zu finden sein, der dem Status quo widerspricht. Singuläres kann sich auch in Figuren des Paradoxes oder des Widerstreits dem schlicht Verstehbaren und Wiederholbaren entziehen. Doch in jedem Fall bleibt dieses Singuläre in Relation zu einem Wiederholbaren, von dem es sich etwa kritisch oder ästhetisch absetzt, wie im Folgenden argumentiert wird. 
positionen, von denen aus individuelle Differenzen überhaupt erst als Differenz ins Auge fallen. Kant sprach in diesem Zusammenhang von dem "Beharrlichen" als Voraussetzung der Wahrnehmung (Kritik der reinen Vernunft). Das heißt, dass das Einzelne (Singuläre etc.) auf das Allgemeinere angewiesen ist. Umgekehrt gilt sicherlich auch, dass das Allgemeine, Wiederholbare sich erst über die Einzelfälle konstituiert.

Mithin sind Einzelnes und Wiederholbares immer schon aufeinander verwiesen. Das gilt auch für den Fall, dass das Einzelne als singuläre Unterbrechung verstanden wird. Entsprechend versuchen geisteswissenschaftliche ebenso wie empirische Arbeiten eine Auseinandersortierung dieser Kategorien mit jeweils unterschiedlicher Tendenz, aber stets auch in Bezug auf die andere Seite. Es wäre daher ein Kurzschluss, wenn man diese Betonung des Wiederholbaren auf der einen Seite oder des Singulären auf der anderen Seite direkt in ein We stem übersetzt. Eher geht es um Steuerungen der Aufmerksamkeit und Feintunıgkeit gegenüber dem Einzelnen oder dem Wiederholbaren. Gerade deshalb ist die vehemente Ablehnung des Allgemeinen, Wiederholbaren, Empirischen durch viele Geisteswissenschaftler problematisch. Historisch mag in dieser Ablehnung das Erbe der kritischen Theorie mit ihrer Aufwertung des `Subversiven` fortwirken. Doch selbst das singuläre NichtFunktionieren, der `Sand im Getriebe , entsteht als Kontrast zum Allgemeinen und Wiederholbaren.

Man kann diagnostizieren, dass die Geisteswissenschaften ein Wiederholbarkertst problem haben. Doch Wiederholbarkeit ist nicht notwendig ein Problem. Während die Energie des geisteswissenschaftlichen Denkens (seit der Abwendung von den Regelpoetiken im achtzehnten Jahrhundert) meist der Abweichung gilt, kann auch das direkte Aufspüren des Wiederholbaren in ihren Bereich fallen. Ästhetik ebenso wie die kulturprägenden Kräfte der Imagination, Narration und Fiktion sind ohne wiederholbare, allgemeine Standards nicht zu denken.

Das Aufspüren des Wiederholbaren erlaubt die Standards von kulturellem Verhalten und ästhetischem Empfinden zu erkennen, auf die wir (oder eine je zu bestimmende Gruppe) gepolt sind - meist ohne es zu wissen, und die unser Handeln im weiten Sinne beeinflussen. Besonders interessant sind diese Standards, wenn sie von Erwartungen abweichen, die man hat oder haben könnte, wie etwa die Erwartung, dass Verhalten rational, gerecht oder gut ist. In der Psychologie wird dafür der Begriff des Bias verwendet. In diesem Punkt stehen sich das Wiederholbare und die Ideologien, die unser Verhalten leiten, nahe. Es genügt sicher nicht, die Ideologien zu kennen, um ihnen zu entgehen, ${ }^{40}$ doch wer sie ni kennt, ist sicher größerer Gefahr ausgesetzt. Als ebenfalls hochinteressant er sich die Suche nach Standards dann, wenn der Standard nicht bekannt ist, also das Gemeinsame, was sich in vielfachen Praktiken zeigt. Beispiele für letzteres sind die Standards, mit dem wir moralische Urteile intuitiv fällen, ${ }^{41}$ oder das Gemeinsame

40 Siehe Paul de Man, Aesthetic ideology, hrsg. Andrzej Warminski, Minneapolis [u. a.] 1996.

41 Jonathan Haidt/Selin Kesebir, »Morality«, in: Fiske/Gilbert/Lindzey (Anm. 23), Bd. 2, 797832 . 
in der ästhetischen Erfahrung ${ }^{42}$. Ein anderer Ausdruck für diese Standards des Wiederholbaren ist der der einfachen Form. Die Suche nach ihr mit allen und eben auch experimentellen Mitteln sollte durchaus mit den Geisteswissenschaften in Einklang stehen. Die einfache Form wie überhaupt das Einfache ist in dieser Hinsicht das Wiederholbare.

\section{Literatur}

Barrett, J. L./Nyhof, M. A. »Spreading non-natural concepts: The role of intuitive conceptual structures in memory transmission of cultural materials«, in: Journal of Cognition and Culture 1 (2001), 69-100.

Bartlett, Frederic C., Remembering: An experimental and social study, Cambridge, Mass. 1932.

Beecher, Donald, »Suspense«, in: Philosophy and Literature 33 (2007), 255-27.

Booker, Christopher, The seven basic plots: Why we tell stories, London/New York 2004.

Breithaupt, Fritz, Kultur der Ausrede. Eine Erzähltheorie, Berlin 2012.

Breithaupt, Fritz/Brower, Eleanor/Whaley, Sarah, »Optimal eventfulness of narratives «, in: Computer Models of Narrative (2015), open access.

Breithaupt, Fritz/Binyan Li/Brower, Eleanor u. a., »Serial reproduction of narratives: Surprise beats facts in event retelling " [in Vorbereitung].

Bruner, Jerome, Making stories. Law, literature, life, Cambridge, Mass. 2002.

Burgoon, J. K., »Interpersonal expectations, expectancy violations, and emotional communication «, in: Journal of Language and Social Psychology 12 (1993), 30-48.

Christianson, S.-Å., »Emotional stress and eyewitness memory: A critical review «, in: Psychological Bulletin 112 (1992), 284-309.

Dudukovic, N. M./Marsh, E. J./Tversky, B., »Telling a story or telling it straight: The effects of entertaining versus accurate retellings on memory«, in: Applied Cognitive Psychology 18 (2004), 125-143.

Campbell, Joseph, The hero with thousand faces [1949], Novato 2008.

Finlayson, Mark A., Learning narrative structure from annotated folktales, $\mathrm{PhD}$ diss., Massachusetts Institute of Technology, Cambridge, Mass. 2012.

Fisseni, Bernhard/Löwe, Benedikt, »Event mapping for comparing formal frameworks for narratives «, in: Logique et Analyse 57 (2014), 181-222.

Flesch, William, Comeuppance. Costly signaling, altruistic punishment, and other biological components of fiction, Cambridge, Mass./London 2007.

Gottschall, Jonathan, The storytelling animal: How stories make us human, Boston, Mass. 2012.

Graffam, J./Turner, J. L., »Escape from boredom: The meaning of eventfulness in the lives of clients at a sheltered workshop «, in: Robert B. Edgerton (Hrsg.), Lives in process: Mildly retarded adults in a large city, Washington, DC 1984, 121-144.

Green, Melanie C./Brock, Timothy C., »The role of transportation in the persuasiveness of public narratives«, in: Journal of Personality and Social Psychology 79 (2000), 701-721.

Green, Melanie C./Donahue, J. K., »Simulated worlds: Transportation into narratives«, in: Keith D. Markman/William M. P. Klein/Julie A. Suhr (Hrsg.), Handbook of imagination and mental simulation, New York 2009, 241-256.

Grimes-Maguire, R./Keane, M. T., »Expecting a surprise? The effect of expectations on perceived surprise in stories", in: Proceedings of the 27th Annual Conference of the Cognitive Science Society (2005), 833-838.

Gruber, Carola, Ereignisse in aller Kürze. Narratologische Untersuchungen zur Ereignishaftigkeit in Kürzestprosa von Thomas Bernhard, Ror Wolf und Helmut Heißenbüttel, Bielefeld 2014.

Haidt, Jonathan/Kesebir, Selin, »Morality«, in: Susan T. Fiske/Daniel T. Gilbert/Gardner Lindzey (Hrsg.), Handbook of Social Psychology, Hoboken, NJ 52010, Bd. 2, 797-832.

42 W. Menninghaus/V. $\Omega$ r/J. Hanich u. a., »Towards a psychological construct of being moved «, in: PloS one 10/6 (2015), e012 8451. 
Hamilton, Andrew/Breithaupt, Fritz, »These things called event: Toward a unified narrative theory of events«, in: Sprache und Datenverarbeitung 37 (2013), 65-87.

Hanich, J./Wagner, V./Shah, M. u. a., »Why we like to watch sad films. The pleasure of being moved in aesthetic experiences", in: Psychology of Aesthetics, Creativity, and the Arts 8 (2014),130-143.

Hühn, Peter, »Event and eventfulness«, in: Peter Hühn/John Pier/Wolf Schmid u. a. (Hrsg.), Handbook of narratology, Berlin/New York 2009, 80-97.

Jolles, André, Einfache Formen. Legende, Sage, Mythe, Rätsel, Spruch, Kasus, Memorabile, Märchen, Witz, Halle (Saale) 1930.

Kahneman, D./Miller, D. T., »Norm theory: Comparing reality to its alternatives«, in: Psychological Review 93 (1986), 136-153.

Kashima, Yoshihisa, »Maintaining cultural stereotypes in the serial reproduction of narratives«, in: Personality and Social Psychology Bulletin 26/5 (2000), 594-604.

Kashima, Yoshihisa/Yeung, Victoria Wai-Lan, »Serial reproduction: An experimental simulation of cultural dynamics«, in: Acta Psychologica Sinica 42/1 (2010), 56-71.

Keen, Susanne, Empathy and the novel, Oxford/New York 2007.

Kensinger, E. A./Corkin, S., »Memory enhancement for emotional words: Are emotional words more vividly remembered than neutral words? «, in: Memory \& Cognition 31 (2003), $1169-1180$

Koriat, A./Goldsmith, M./Pansky, A., »Toward a psychology of memory accuracy«, in: Annual review of psychology 51 (2000), 481-537.

Koschorke, Albrecht, Wahrheit und Erfindung: Grundzüge einer Allgemeinen Erzähltheorie, Frankfurt a. M. 2012.

Lyons, Anthony/Kashima, Yoshihisa, "Maintaining stereotypes in communication: Investigating memory biases and coherence-seeking in storytelling", in: Asian Journal of Social Psychology 9/1 (2006), 59-71.

Maguire, R./Maguire, P./Keane, M. T., "Making sense of surprise: an investigation of the factors influencing surprise judgments «, in: Journal of Experimental Psychology: Learning, Memory, and Cognition 37 (2011), 176-186.

Man, Paul de, Aesthetic ideology, hrsg. Andrzej Warminski, Minneapolis [u. a.] 1996.

Margulis, Elizabeth H., On repeat: How music plays the mind, Oxford 2014.

Marsh, Elizabeth J., »Retelling is not the same as recalling: Implications for memory«, in: Current Directions in Psychological Science 16 (2007), 16-20.

Menninghaus, W./Wagner, V./Hanich, J. u. a., »Towards a psychological construct of being moved «, in: PloS one 10 (2015), e012 8451.

Moussaïd, Mehdi/Brighton, Henry/Gaissmaier, Wolfgang, »The amplification of risk in experimental diffusion chains «, in: Proceedings of the National Academy of Sciences 112/18 (2015), 5631-5636.

Nabi, R. L./Green, M. C., »The role of a narrative's emotional flow in promoting persuasive outcomes«, in: Media Psychology 18 (2015), 137-162.

Norenzayan, Ara/Atran, Scott/Faulkner, Jason u. a., "Memory and mystery: The cultural selection of minimally counterintuitive narratives«, in: Cognitive Science 30/3 (2006), 531-553.

Polti, Georges, Les Trente-Six Situations Dramatiques, Paris 1895.

Prince, Gerald, »Narrativehood, narrativity, narratability«, in: John Pier/José Ángel García Landa (Hrsg.), Theorizing narrativity, Berlin/New York 2008, 19-27.

Propp, Wladimir, Morphologie des Märchens [1928], München 1972.

Radvansky, Gabriel A./Zachs, Jeffrey M., Event cognition, Oxford 2014.

Rapp, David N./Gerrig, Richard J., "Predilections for narrative outcomes: The impact of story contexts and reader preferences«, in: Journal of Memory and Language 54 (2006), 54-67.

Reisenzein, Rainer, »Exploring the strength of association between components of emotion syndromes: The case of surprise«, in: Cognition \& Emotion 10 (2000), 241-277.

Schmid, Wolf, »Narrativity and eventfulness «, in: Tom Kindt/Hans-Harald Müller (Hrsg.), What is narratology? Questions and answers regarding the status of a theory, Berlin/New York 2003, 17-35.

Schmid, Wolf, Narratology. An introduction, Berlin/New York 2010. 
Silva, Paul J., »Looking past pleasure: Anger, confusion, disgust, pride, surprise, and other unusual aesthetic emotions«, in: Psychology of Aesthetics, Creativity, and the Arts 3 (2009), $48-51$.

Teigen, K. H./Keren, G., »Surprises: Low probabilities or high contrasts? «, in: Cognition 87 (2003), 55-71.

Voss, Christiane, Narrative Emotionen. Eine Untersuchung über Möglichkeiten und Grenzen philosophischer Emotionstheorien, Berlin/New York 2004.

Welzer, H., »Re-narrations: How pasts change in conversational remembering «, in: Memory studies 3 (2010), 5-17.

Winograd, Eugene/Neisser, Ulric (Hrsg.), Affect and accuracy in recall: Studies of flashbulb< memories, Cambridge, Mass. 2006. 